\section{ORIGINAL RESEARCH}

\author{
P.J. MacMahon \\ M.J. Shelly \\ D. Scholz \\ S.J. Eustace \\ E.C. Kavanagh
}

\title{
Injectable Corticosteroid Preparations: An Embolic Risk Assessment by Static and Dynamic Microscopic Analysis
}

\begin{abstract}
BACKGROUND AND PURPOSE: Transforaminal CS injections have been associated with severe adverse CNS events, including brain and spinal cord infarction. Our purpose was to describe the static and dynamic microscopic appearances of CS preparations, with an emphasis on their potential to cause adverse central nervous system events by embolic mechanisms during transforaminal injection.
\end{abstract}

\begin{abstract}
MATERIALS AND METHODS: Pharmaceutical preparations of nondilute injectable CSs were used after appropriate mixing: MPA (40 mg/mL), TA (40 mg/mL), and DSP (8 mg/2 mL). For dynamic imaging, a novel methodology was devised to replicate the flow of crystals within spinal cord arterioles. In addition, CS preparations were mixed with plasma to assess for changes in crystal size, morphology, and tendency to aggregate.
\end{abstract}

\begin{abstract}
RESULTS: The CS preparations MPA and TA are composed of crystals of varying sizes. MPA crystal size range was 0.4-26 $\mu \mathrm{m}$ (mean, $6.94 \mu \mathrm{m}$ ), TA crystal size range 0.5-110 $\mu \mathrm{m}$ (mean, $17.4 \mu \mathrm{m}$ ), and DSP did not contain any significant crystals or particles. There was no change in the crystal morphology or propensity to aggregate after mixing with local anesthetic. After mixing with plasma, the crystals also were unchanged; however, there was a significant reduction in the size of aggregates. On dynamic imaging, these aggregates were proved to maintain their integrity and to act as potential embolization agents.
\end{abstract}

CONCLUSIONS: MPA and TA have a substantial risk of causing infarction by embolization if inadvertently injected intra-arterially at the time of TFESI. DSP is completely soluble and microscopically has no potential to obstruct arterioles. When performing cervical TFESI procedures, the administration of insoluble CSs should be avoided.

ABBREVIATIONS: CNS = central nervous system; $C S=$ corticosteroid; DSP $=$ dexamethasone sodium phosphate; $L A=$ local anesthetic; $M P A=$ methylprednisolone acetate; $R B C$ - red blood cell; TA = triamcinolone acetonide; TFESI = transforaminal epidural spinal injection

Ins njectable corticosteroid preparations are routinely administered to treat various inflammatory disorders of the body. In general, their mode of action and potential to cause adverse effects are well established. ${ }^{1}$

In the recent literature, there has been considerable controversy with regard to the severity of adverse effects associated with transforaminal steroid injections (also referred to as selective nerve root blocks), especially those performed at the level of the cervical spine. These procedures are routinely performed to treat radicular symptoms by administering CS and LA into the region of the neural foramen. Unfortunately, this injection technique has been associated with severe adverse CNS events, with brain and spinal cord infarction being the most frequent of these uncommon sequelae. ${ }^{2-7}$ At present, there is some debate in

Received January 9, 2011; accepted after revision February 20.

From the Department of Radiology (P.J.M., M.J.S., S.J.E., E.C.K.), Mater Misericordiae University Hospital, Dublin, Ireland; and Conway Institute (D.S.), University College, Dublin, Ireland.

Paper previously presented at: Radiological Society of North America Annual Meeting, November 28-December 3, 2010, Chicago, Illinois.

Please address correspondence to Peter J. MacMahon, MD, Department of Musculoskeletal Imaging and Intervention, Massachusetts General Hospital, YAW 6030, Boston, MA 02114; e-mail: petermacmahon@yahoo.com

Indicates article with supplemental on-line figures.

Indicates article with supplemental on-line videos.

http://dx.doi.org/10.3174/ajnr.A2656 the literature as to the exact etiology of these adverse events. Postulated causes include 1) spinal cord or cerebral embolic infarction after particulate CS injection into an arterial vessel; 2) vascular injury causing arterial spasm, trauma, or compression; and 3) neurotoxicity from the preservative, drug vehicle, or both in the CS formulation. ${ }^{1}$

Although it is generally accepted that the most likely etiology of the observed events is embolization of arterioles by particulate CSs leading to CNS infarction, ${ }^{2,8,9}$ a recent in vivo study has suggested that the observed effects may, at least in part, be explained by nonembolization mechanisms. ${ }^{10}$ The authors of this 2009 paper, similarly to researchers of other studies, ${ }^{11-13}$ have suggested that substantial CNS injury can be caused by the components found in commercial CS formulations, namely, benzyl alcohol and polyethylene glycol. In addition, this paper demonstrated CNS injury after the intra-arterial injection of a nonparticulate $\mathrm{CS}$, suggesting that CSs have direct toxic effects on the CNS. This has raised doubts as to the role of particulate CSs in the observed adverse CNS events and thus whether the adverse events are actually related to nonembolization-type phenomena. ${ }^{14}$

The aim of this in vitro study was to assess the capacity of the commonly used CS preparations to cause embolic phenomenon via microscopic analysis. CSs were assessed before and after mixing with both LA and human plasma. In addition, we performed a dynamic assessment with video microscopy as preparations flowed through a $200-\mu \mathrm{m}$ depth channel to simulate passage through a CNS arteriole. 


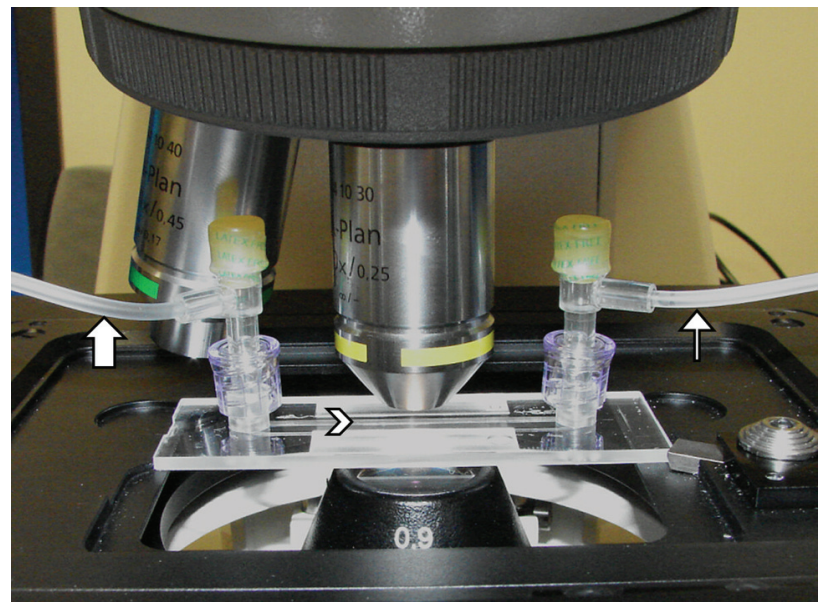

Fig 1. Photograph of the apparatus within the microscope. Samples were injected through tubing (wide arrow) into the $\mu$-Slide device (arrowhead demonstrates direction of flow through the channel within the device) and then exit into effluent tubing (thin arrow).

\section{Materials and Methods}

\section{Agents}

The analyzed CS preparations were limited to $40 \mathrm{mg} / \mathrm{mL}$ MPA (DepoMedrol; Pfizer, New York, New York), 40 mg/mL TA (Kenalog; Bristol-Myers Squibb, New York, New York), and $4 \mathrm{mg} / \mathrm{mL}$ DSP (Hospira, Lake Forest, Illinois). The LA preparation used was $0.25 \%$ bupivacaine hydrochloride (Marcaine; Astrazeneca, London, United Kingdom). Human plasma was obtained by centrifuging whole blood at $3000 \mathrm{rpm}$ for 10 minutes. Plasma was obtained by using a disposable pipette.

\section{Materials}

At all stages standard syringes and needles were used to replicate clinically performed CS preparation and administration techniques. The smallest caliber needle used was a 25-gauge needle. We used the $\mu$-Slide I Luer flow kit (Ibidi, Munich, Germany) to perform dynamic microscopic analysis. This allows microscopic images to be obtained as CS preparations flow through a $200-\mu$ m-depth channel (Fig 1).

For all static imaging, measurements were obtained with an Axioskop 40 microscope (Carl Zeiss Microimaging, Jena, Germany) fitted with an OptiScan motorized stage (Prior Scientific, Cambridge, United Kingdom) controlled by the Image-Pro Plus 6 image analysis program (Media Cybernetics, Bethesda, Maryland). Images were collected by using an Achroplan $\times 10, \times 20$, and $\times 40$ phase contrast objective with a ProgRes $\mathrm{C} 10+$ digital camera (Jenoptik Ag, Jena, Germany). The images were calibrated for each objective on the microscope by using the stage movement controlled by the Image-Pro Plus Scope Pro plug-in. These calibrations were checked against a slide micrometer.

For all dynamic imaging, an AxioCam HR charge-coupled device camera in black-and-white mode (Carl Zeiss Microimaging) was used at $1388 \times 1040$ resolution, 12 images/s, and videos recorded via Axiovision software (version 4.7; Carl Zeiss Microimaging).

\section{Methods}

All agents were shaken as to manufacturer's instructions. Nine separate samples were prepared for analysis: MPA only, TA only, DSP only, MPA + LA, TA + LA, DSP + LA, MPA + LA + plasma, TA + $\mathrm{LA}+$ plasma, and DSP + LA + plasma (Table). A mixture ratio of 1:1 or 1:1:1 was used for the prepared samples. For every $1 \mathrm{~mL}$ (mil) of CS,
1 mil of LA was mixed (1:1). All samples were prepared immediately before imaging (delay of no $>2$ minutes between preparation and imaging).

For static imaging, a single drop of prepared sample was placed on a microscope slide via a standard 25 -gauge needle, and a coverslip was applied. High resolution $(2080 \times 1542)$ color RGB digital images were then obtained at $\times 100, \times 200$, and $\times 400$ magnification after calibration had been performed. For dynamic imaging, the prepared samples were manually injected via a short low-pressure tube attached to the $\mu$-Slide device (Fig 1). Injection speed was similar to standard clinical technique. Gray-scale digital video clips by using MPEG4 encoding at a resolution of $1360 \times 1040,10$ frames/s, and data rate of $2648.59 \mathrm{kbits} / \mathrm{s}$ were then obtained. All video clips were calibrated and annotated. All dynamic imaging was performed at $\times 200$ magnification only.

For static measurements, random fields of view of each preparation were used. From the high magnification $(\times 400)$ images, individual crystals were measured to establish minimum, maximum, and mean sizes. Lower power magnification $(\times 100)$ images were used to measure minimum, maximum, and mean sizes of the crystal aggregates. On dynamic imaging, random frames from the video clip were used to obtain the minimum, maximum, and mean crystal aggregate measurements.

A size cutoff of $10 \mu \mathrm{m}$ was used for group analysis. This number was picked because particles $>10 \mu \mathrm{m}$ have been demonstrated to occlude capillaries in vivo. ${ }^{15}$ It is also of note that the average diameter of a $\mathrm{RBC}$ is $6-8 \mu \mathrm{m}$.

StatPlus (AnalystSoft, Alexandria, Virginia) was used for statisti$\mathrm{cal}$ analysis. The Student $t$ test was used to test for statistical significance. This study was exempted from Institutional Review Board approval.

\section{Results}

\section{$M P A$}

MPA was confirmed to be a crystalline preparation (On-line Fig 1). Crystals tend to be oval or round and relatively uniform in size (mean, $6.9 \mu \mathrm{m}$; range, $0.5-26 \mu \mathrm{m}$ ). In any random field of view, most crystals were less than the size of a RBC (On-line Fig 2). A mean of $16.7 \%$ of crystals were $>10 \mu \mathrm{m}$ (Table). On static imaging the crystals tended to aggregate (On-line Fig 1). Approximately $84.7 \%$ of crystals were aggregated into larger particles. Aggregates ranged from 5 to $200 \mu \mathrm{m}$, with a mean size of $59 \mu \mathrm{m}$, when formed on a glass slide. Mixing with LA had no significant effect on crystal sizes or aggregation (Online Fig 3). Mixing with plasma, however, caused a reduction in the size of the visualized aggregates (mean, $21.6 \mu \mathrm{m}$; range, 5-55 $\mu \mathrm{m}$; On-line Fig 4). There was no change in the size of individual crystals after mixing with plasma (On-line Fig 5).

On dynamic imaging, the aggregation of crystals was confirmed to be real and not an artifact of using glass slides. The aggregates visualized flowing through the channel ranged from 5 to $195 \mu \mathrm{m}$, with a mean of $42.9 \mu \mathrm{m}$. These aggregates maintained their integrity during injection and hence effectively act as large particles (On-line Fig 6). Similar to the results of static imaging, there was no change in aggregation or crystal size after mixing with local anesthetic (On-line Video 1). After mixing with plasma, aggregates significantly reduced in size. These microaggregates ranged from 5 to $50 \mu \mathrm{m}$, with a mean size of $21.6 \mu \mathrm{m}$ (Fig 2). The injectate appeared denser 


\begin{tabular}{|c|c|c|c|c|c|c|}
\hline \multicolumn{7}{|c|}{ Comparison of the crystals and aggregates found in MPA and TA at high-powered microscopy } \\
\hline & MPA & MPA + LA & MPA + LA + Plasma & TA & $\mathrm{TA}+\mathrm{LA}$ & $\mathrm{TA}+\mathrm{LA}+$ Plasma \\
\hline \multicolumn{7}{|l|}{ Crystal size } \\
\hline Mean $(\mu \mathrm{m})$ & 6.94 & 7.1 & 6.91 & 17.4 & 17.1 & 17.9 \\
\hline Range $(\mu \mathrm{m})$ & $0.5-26$ & $0.4-26$ & $0.5-25$ & $1-110$ & $0.5-108$ & $0.3-110$ \\
\hline$\%>10 \mu \mathrm{m}^{\mathrm{a}}$ & 16.7 & 15.9 & 17.1 & 38 & 41 & 40 \\
\hline$\%$ aggregated $^{\mathrm{b}}$ & $85^{c}$ & 83 & $70^{c, *}$ & $85^{c}$ & 81 & $10^{c, *}$ \\
\hline \multicolumn{7}{|l|}{ Aggregate size } \\
\hline Mean $(\mu \mathrm{m})$ & $42.9^{c}$ & 45.1 & $21.64^{\mathrm{c}, *}$ & $14.3^{\mathrm{C}}$ & 15.1 & $9.8^{\mathrm{c}, *}$ \\
\hline Range $(\mu \mathrm{m})$ & 5-195 & $4.1-186$ & $3.2-50$ & $2-115$ & $3-111$ & $5-25$ \\
\hline$\%>10 \mu \mathrm{m}^{\mathrm{d}}$ & 88 & 82 & 80 & $28^{\mathrm{c}}$ & 22 & $40^{\mathrm{c}, *}$ \\
\hline
\end{tabular}

Note:-DSP is not included in this table as there were no crystals or aggregates identified in DSP.

a Percentage of crystals $>10 \mu \mathrm{m}$ and therefore with embolic potential.

b Percentage of crystals that are formed into aggregates.

${ }^{c}$ Denoting where there were statistically significant differences in measurements between plasma and nonplasma mixed corticosteroid $(P<.05)$.

${ }^{\mathrm{d}}$ Percentage of crystal aggregates that are $>10 \mu \mathrm{m}$ and therefore with embolic potential.

* Denoting where differences in measurement were statistically significant between MPA and TA.

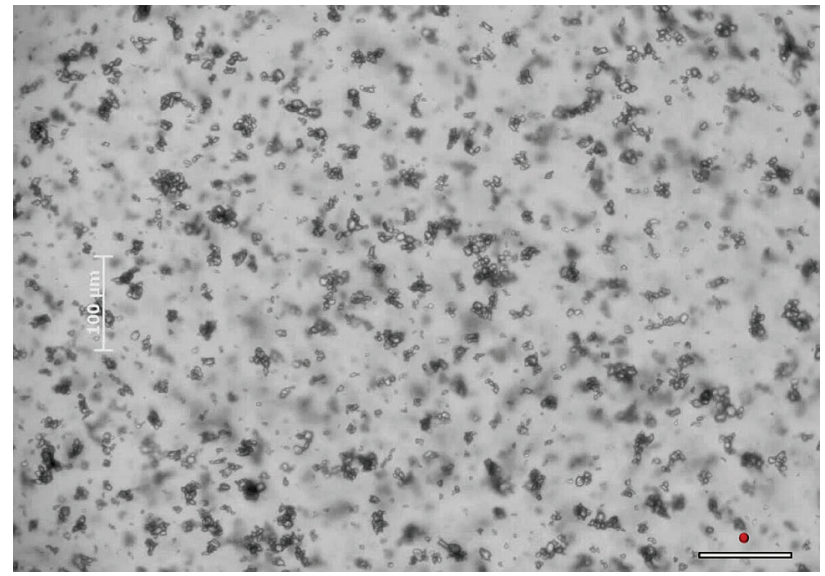

Fig 2. Freeze frame from On-line Video 2 of MPA flowing through a 200- $\mu$ m-depth channel after mixing with LA and plasma. This demonstrates the reduction in size of MPA aggregates after mixing with plasma. The white scale bar in the bottom right of the image represents $100 \mu \mathrm{m}$. The adjacent red circle represents the average size of a RBC at this magnification. There is a separate scale bar on the left side of the image that is somewhat obscured.

due to the more diffuse dispersion of individual crystals and smaller aggregates (On-line Video 2). Approximately $80 \%$ of aggregates, after mixing with plasma, were $>10 \mu \mathrm{m}$ as visualized on dynamic imaging.

\section{$T A$}

TA is also a crystalline preparation; however, the crystals are morphologically different to MPA (On-line Fig 7). The largest crystals tended to be rectangular (On-line Fig 8). There is also a greater range in the size of TA crystals compared with MPA. Individual crystals ranged in size from 1 to $110 \mu \mathrm{m}$, with a mean size of $17.4 \mu \mathrm{m}$. Approximately $38 \%$ of crystals were $>10 \mu \mathrm{m}$ in size. Similar to MPA approximately $85 \%$ of crystals were components of aggregates; however, the largest crystals did not tend to aggregate together. As a result, aggregates measured no $>15 \mu \mathrm{m}$. Most aggregates were much smaller than this and composed of small crystals. Like MPA, there was no effect after mixing with LA. After mixing with plasma, crystal aggregation reduced to an even greater degree than that seen with MPA (On-line Fig 9).

On dynamic imaging, the integrity of the large individual crystals and crystal aggregates was unchanged. There was no change on mixing with LA (On-line Fig 10 and On-line Video 3). After mixing with plasma, very few aggregates were visual-

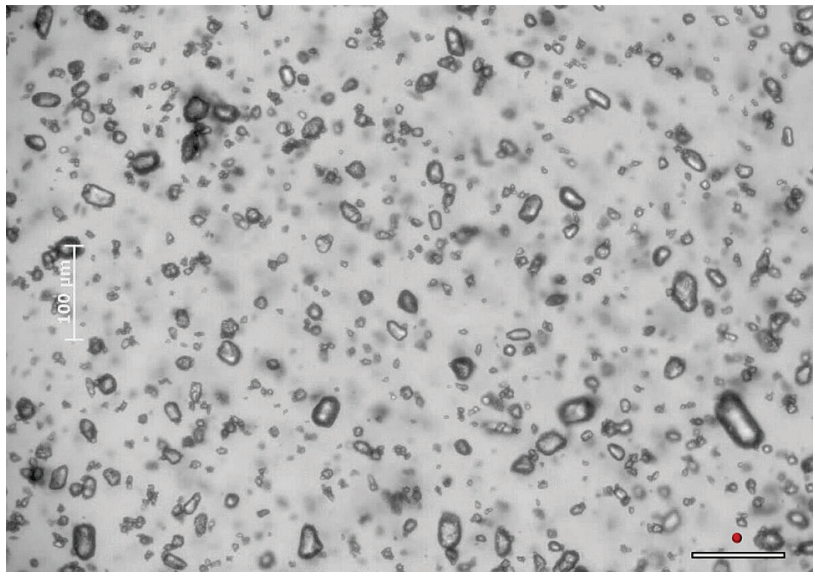

Fig 3. Freeze frame from On-line Video 4 of TA flowing through a 200- $\mu \mathrm{m}$-depth channel after mixing with LA and plasma. This demonstrates the near complete absence of crystal aggregation after mixing with plasma. The white scale bar in the bottom right of the image represents $100 \mu \mathrm{m}$. The adjacent red circle represents the average size of a RBC at this magnification. There is a separate scale bar on the left side of the image.

ized (Fig 3 and On-line Video 4). Those that were present had a range of sizes from 5 to $25 \mu \mathrm{m}$, with a mean of $9.8 \mu \mathrm{m}$. Approximately $40 \%$ of aggregates measured $>10 \mu \mathrm{m}$. Overall, approximately $50 \%$ of the "particulates" (ie, crystals or aggregates) in TA when mixed with plasma are $>10 \mu \mathrm{m}$.

\section{DSP}

DSP is not a crystalline preparation (On-line Fig 11). Because the environment in which the DSP samples were prepared was not perfectly clean, small skin cells and other tiny particles (submicrometer in size) were occasionally seen on high magnification. Otherwise, no crystals or particulates $>1 \mu \mathrm{m}$ were identified at high magnification on static imaging. Certainly, there is no constituent of DSP close to, or larger than, a RBC. Mixing with LA had no effect, and no particulates were precipitated. Mixing with plasma revealed the presence of a few RBCs remaining after centrifuging of whole blood but no change to the DSP preparation.

On dynamic imaging, there was no change to these results (On-line Video 5 and Fig 4). The residual RBCs were visible flowing through the channel (On-line Video 6).

\section{Discussion}

The recent reporting of severe adverse CNS events, including death, occurring during or immediately after TFESI proce- 


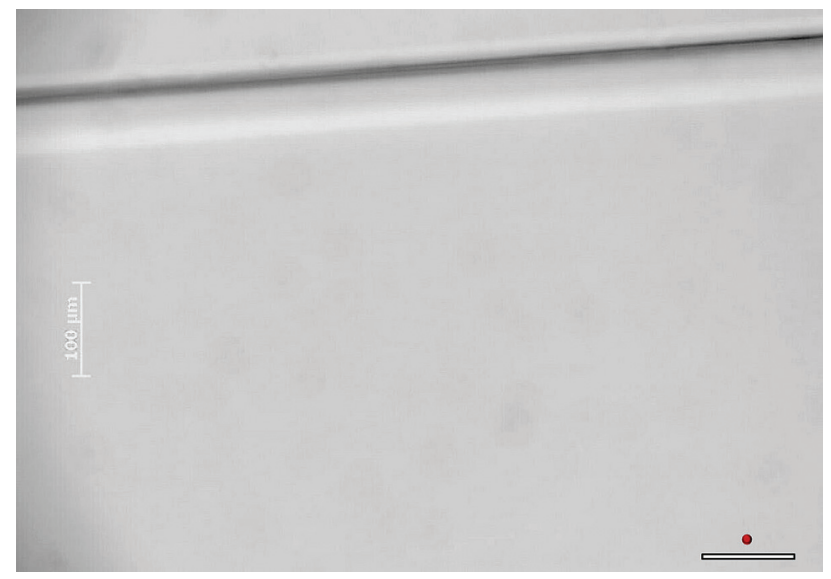

Fig 4. Freeze frame from On-line Video 5 of DSP flowing through a 200- $\mu$ m-depth channel after mixing with LA and plasma. The straight line at the top of the image is the edge of the channel. No crystals or significant particulates are identified. The white scale bar in the bottom right of the image represents $100 \mu \mathrm{m}$. The adjacent red circle represents the average size of a RBC at this magnification. There is a separate scale bar on the left side of the image.

dures has prompted detailed assessment of the potential mechanisms by which these events have transpired. It is now generally accepted that inadvertent injection of the CS-LA mixture into a radicular artery supplying the CNS is the most likely explanation in most of the reported events. This has led to detailed examination of the constituents of the injectate, with most emphasis on the composition of CSs.

Commercially available CSs can be divided into 3 groups: insoluble (includes MPA and TA), soluble (includes DSP), and a formulation composed of a mixture of insoluble and soluble CSs. ${ }^{1}$ In general, injectates containing insoluble CSs have been preferred over soluble CSs during TFESI procedures because a medium- to long-term relief of symptoms is desired. ${ }^{1,16,17}$ This long-term benefit principally derives from the fact that insoluble CSs are esters and hence require cellular esterases for the active steroid moiety to be released. In addition, insoluble CS preparations are supplied as a crystalline powder in aqueous suspension that is less likely to be systemically absorbed soon after injection compared with soluble preparations. ${ }^{17}$ It is this crystalline nature of insoluble CSs, with the potential to occlude arterioles, that is now regarded as the leading cause of the reported CNS adverse events occurring during TFESI. It is important to note that there are no case reports of events occurring with the use of soluble CSs.

Researchers have previously examined CSs by microscopy to evaluate their crystalline appearance. ${ }^{18-20}$ The clarity of the images obtained and the analysis of only static preparations, however, limited the ability to fully clarify their embolization potential. Our study has used higher powered, higher resolution microscopes in combination with close replication of clinical procedures and the dynamic intra-arterial environment to analyze the propensity of CSs to cause embolization events.

The results of this study demonstrate that the 2 most commonly administered insoluble CSs-MPA and TAare composed of individual crystals that range in sizes significantly larger than RBCs; $16.7 \%$ of MPA crystals and $38 \%$ of TA crystals are $>10 \mu \mathrm{m}$. In addition, these crystals do not dissolve or change in morphology after mixing with LA or plasma.

Importantly, as has been suggested in other papers, insoluble CS crystals can form larger aggregates when dropped onto a static microscope slide. ${ }^{18}$ We have demonstrated that these apparent aggregates maintain their integrity when flowing through an arteriole simulator and therefore act as a larger particulate in the arterial system. Interestingly, after mixing with plasma, these aggregates significantly decrease in size. The mean sizes of MPA crystal aggregates before and after mixing with plasma were 42.9 and $21.64 \mu \mathrm{m}$, respectively $(P<.05)$. Similarly for TA, the mean sizes of crystal aggregates before and after mixing with plasma were 14.3 and $9.8 \mu \mathrm{m}$, respectively $(P<.05)$. Crystal aggregation in TA was so inhibited after mixing with plasma that it was thought unlikely to play a major role in any potential embolization events. TA crystals tend to be quite large (maximum size of $110 \mu \mathrm{m}$ ) and are thus capable of occluding tiny vessels. It is not clear why crystal aggregation is inhibited when mixed with plasma but it is possibly similar to the mechanism by which calcium oxalate crystal aggregation in urine is inhibited by serum albumin and globulins. ${ }^{21}$ Plasma contains albumin and globulins that may have inhibitory effects on individual intercrystal adhesion, thereby reducing the size of the aggregates that form.

DSP was confirmed to be a completely soluble form of CS without evidence of any crystalline structures at high-powered microscopy. In addition, there was no change to the appearance of DSP after mixing with LA and plasma.

The clinical significance of these results is readily evident. Insoluble CSs are composed of crystals and larger aggregates, capable of occluding the small end arterioles supplying the CNS; thus, they present an extremely high risk of causing infarction. Indeed, the fact that insoluble CSs, when mixed with plasma, are composed of smaller aggregates (still $>10 \mu \mathrm{m})$ than reported previously, increases the risk of infarction as arterial injection will generate a shower of small particulates that will obstruct the most distal arterioles where collateral vessels are less likely to be present. ${ }^{22}$ DSP cannot cause embolization events because it does not contain structures that are larger than the size of RBCs. It is important to note that these results are not only applicable to scenarios involving paraspinal injections but also relevant to any scenario where inadvertent injection of CSs is possible, especially at sites with limited ability to collateralize or cope with short-term distal microembolization (eg, injections at the wrist or ankle).

These results are compatible with an in vivo study performed on pigs examining the effect of direct vertebral artery injection of insoluble CSs versus soluble CSs. ${ }^{23} \mathrm{We}$ demonstrated that DSP had no effect on pigs as assessed by functional examination, imaging, and histologic examination. Conversely, the administered insoluble CS (MPA) induced visible ischemic changes in the CNS on MR imaging, with eventual death in all cases. These results reinforce the concept that insoluble CSs are extremely dangerous if they enter an artery supplying the CNS.

The most common preservative and drug vehicle present in steroid injectables is benzyl alcohol and polyethylene glycol, respectively. These have been postulated by many studies to be sources of significant toxicity. ${ }^{24-26}$ Neurotoxic effects ascribed to benzyl alcohol include demyelination and neural degener- 
ation. ${ }^{27,28}$ Polyethylene glycol, found in MPA, has been shown to reversibly decrease the action potentials of neural fibers; however, no clear link with adverse neurologic events has been established. ${ }^{29,30}$ TA and DSP formulations do not usually contain polyethylene glycol, but standard formulations do typically contain benzyl alcohol. Because DSP has been demonstrated to be completely safe intra-arterially in several in vivo studies, and it is routinely administered intravenously, it can be reasonably concluded that benzyl alcohol does not play a significant role in the observed neurologic events during TFESI. Benzyl alcohol does not seem to be a completely benign component to steroid formulations, however, and consideration should be given to the use of preservative-free formulations.

It is an extremely rare occurrence to inadvertently puncture an artery associated with the CNS by using standard fluoroscopic transforaminal techniques; however, several studies have demonstrated such incidents. ${ }^{4,31,32}$ In these reports, a radicular artery communicating with the spinal cord is demonstrated to opacify during standard TFESI.

The standard technique is to place the needle tip at the posteroinferior aspect of the cervical foramen. This location is selected specifically because it is a relatively nonvascular region. However, Huntoon ${ }^{33}$ has demonstrated, via very detailed anatomic examination, that 7 of 95 dissected intervertebral foramina had an arterial vessel at the posterior aspect of the foraminal opening that formed radicular or segmental medullary vessels to the spinal cord. This is the likely means by which CSs can be unintentionally injected into an artery supplying the CNS, leading to embolization and infarction. It is likely that at other levels of the spine some patients will have a rare anatomic variant of an arterial vessel that supplies the CNS at the site of injection, thus explaining the case reports of severe CNS adverse events occurring with the use of insoluble CSs during lumbar TFESIs ${ }^{34,35}$ and lumbar interlaminar epidural injections. ${ }^{36,37}$

The limitations of our study are that we did not use whole blood but only assessed mixing with plasma. This was done so that we could visualize the CS crystals. The attenuation of RBCs in whole blood nearly completely obscures evaluation of the crystals and prevents accurate morphologic assessment and measurement. In addition, we did not evaluate CSs after mixing with contrast agents. In most institutions, including ours, contrast is not routinely mixed in the same syringe as the pharmaceutical; thus, we felt it was not a clinically important component to include.

\section{Conclusions}

Insoluble CS preparations have a substantial risk of causing infarction by embolization if inadvertently injected intraarterially at the time of TFESI. DSP, in contrast, is completely soluble and microscopically has no potential to obstruct arterioles. When performing cervical TFESI procedures, the administration of insoluble CSs should be avoided. Indeed when performing any paraspinal injection with insoluble CSs extreme caution should always be exercised because even though the risk of intra-arterial injection is low, the potential complications are devastating.

\section{References}

1. MacMahon PJ, Eustace SJ, Kavanagh EC. Injectable corticosteroid and local anesthetic preparations: a review for radiologists. Radiology 2009;252:647-61

2. Rathmell JP, Aprill C, Bogduk N. Cervical transforaminal injection of steroids. Anesthesiology 2004;100:1595-600

3. Rosenkranz M, Grzyska U, Niesen W, et al. Anterior spinal artery syndrome following periradicular cervical nerve root therapy. J Neurol 2004;251:229-31

4. Brouwers PJ, Kottink EJ, Simon MA, et al. A cervical anterior spinal artery syndrome after diagnostic blockade of the right C6-nerve root. Pain 2001; 91:397-99

5. Ruppen W, Hugli R, Reuss S, et al. Neurological symptoms after cervical transforaminal injection with steroids in a patient with hypoplasia of the vertebral artery. Acta Anaesthesiol Scand 2008;52:165-66

6. Muro K, O'Shaughnessy B, Ganju A. Infarction of the cervical spinal cord following multilevel transforaminal epidural steroid injection: case report and review of the literature. J Spinal Cord Med 2007;30:385-88

7. Suresh S, Berman J, Connell DA. Cerebellar and brainstem infarction as a complication of CT-guided transforaminal cervical nerve root block. Skeletal Radiol 2007;36:449-52

8. Baker R, Dreyfuss P, Mercer S, et al. Cervical transforaminal injection of corticosteroids into a radicular artery: a possible mechanism for spinal cord injury. Pain 2003;103:211-15

9. McMillan MR, Crumpton C. Cortical blindness and neurologic injury complicating cervical transforaminal injection for cervical radiculopathy. Anesthesiology 2003;99:509-11

10. Dawley JD, Moeller-Bertram T, Wallace MS, et al. Intra-arterial injection in the rat brain: evaluation of steroids used for transforaminal epidurals. Spine (Phila Pa 1976) 2009;34:1638-43

11. Macky TA, Helmy D, El Shazly N. Retinal toxicity of triamcinolone's vehicle (benzyl alcohol): an electrophysiologic and electron microscopic study. Graefes Arch Clin Exp Ophthalmol 2007;245:817-24

12. Hahn AF, Feasby TE, Gilbert JJ. Paraparesis following intrathecal chemotherapy. Neurology 1983;33:1032-38

13. Hetherington NJ, Dooley MJ. Potential for patient harm from intrathecal administration of preserved solutions. Med J Aust 2000;173:141-43

14. Rasul Z, Sell P. Lumbar nerve root pain: what works and what doesn't? Int Musculoskeletal Med 2009;31:166-71

15. Gesler RM, Garvin PJ, Klamer B, et al. The biologic effects of polystyrene latex particles administered intravenously to rats-a collaborative study. Bull Parenter Drug Assoc 1973;27:101-13

16. Dent $\mathrm{PB}, \mathrm{Walker} N$. Intra-articular corticosteroids in the treatment of juvenile rheumatoid arthritis. Curr Opin Rheumatol 1998;10:475-80

17. Cole BJ, Schumacher HR Jr. Injectable corticosteroids in modern practice. J Am Acad Orthop Surg 2005;13:37-46

18. Tiso RL, Cutler T, Catania JA, et al. Adverse central nervous system sequelae after selective transforaminal block: the role of corticosteroids. Spine J 2004; $4: 468-74$

19. Derby R, Lee SH, Date ES, et al. Size and aggregation of corticosteroids used for epidural injections. Pain Med 2008;9:227-34

20. Benzon HT. Epidural steroid injections for low back pain and lumbosacral radiculopathy. Pain 1986;24:277-95

21. Grover PK, Moritz RL, Simpson RJ, et al. Inhibition of growth and aggregation of calcium oxalate crystals in vitro-a comparison of four human proteins. Eur J Biochem 1998;253:637-44

22. Sliwa JA, Maclean IC. Ischemic myelopathy: a review of spinal vasculature and related clinical syndromes. Arch Phys Med Rehabil 1992;73:365-72

23. Okubadejo GO, Talcott MR, Schmidt RE, et al. Perils of intravascular methylprednisolone injection into the vertebral artery. An animal study. J Bone Joint Surg Am 2008;90:1932-38

24. Younis HS, Shawer M, Palacio K, et al. An assessment of the ocular safety of inactive excipients following sub-tenon injection in rabbits. J Ocul Pharmacol Ther 2008;24:206-16

25. Chang YS, Wu CL, Tseng SH, et al. In vitro benzyl alcohol cytotoxicity: implications for intravitreal use of triamcinolone acetonide. Exp Eye Res 2008; 86:942-50

26. Toyooka K, Fujimura H. Iatrogenic neuropathies. Curr Opin Neurol 2009; 22:475-79

27. Manchikanti L. Role of neuraxial steroids in interventional pain management. Pain Physician 2002;5:182-99

28. Craig DB, Habib GG. Flaccid paraparesis following obstetrical epidural anesthesia: possible role of benzyl alcohol. Anesth Analg 1977;56:219-21

29. Ray CE. Pain Management in Interventional Radiology. Cambridge, United Kingdom: Cambridge University Press; 2008

30. Spaccarelli KC. Lumbar and caudal epidural corticosteroid injections. Mayo Clinic Proc 1996;71:169-78

31. Yin W, Bogduk N. Retrograde filling of a thoracic spinal artery during transforaminal injection. Pain Med 2009;10:689-92 
32. Verrills $P$, Nowesenitz G, Barnard A. Penetration of a cervical radicular artery during a transforaminal epidural injection. Pain Med 11:229-31

33. Huntoon MA. Anatomy of the cervical intervertebral foramina: vulnerable arteries and ischemic neurologic injuries after transforaminal epidural injections. Pain 2005;117:104-11

34. Houten JK, Errico TJ. Paraplegia after lumbosacral nerve root block: report of three cases. Spine J 2002;2:70-75
35. Kennedy DJ, Dreyfuss P, Aprill CN, et al. Paraplegia following image-guided transforaminal lumbar spine epidural steroid injection: two case reports. Pain Med 2009;10:1389-94

36. Thefenne L, Dubecq C, Zing E, et al. A rare case of paraplegia complicating a lumbar epidural infiltration. Ann Phys Rehabil Med 2010;53:575-83

37. Lenoir T, Deloin X, Dauzac C, et al. Paraplegia after interlaminar epidural steroid injection: a case report. Rev Chir Orthop Reparatrice Appar Mot 2008;94:697-701 